Editorial

\title{
Importance of Selecting Route of Administration in Designing Products for Diabetic Foot Ulcers
}

\author{
Veera Venkata Satyanarayana Reddy Karri*
}

Department of Pharmaceutics, JSS College of Pharmacy, Ootacamund, JSS University, Mysore, India

*Corresponding Author: Veera Venkata Satyanarayana Reddy Karri, Department of Pharmaceutics, JSS College of Pharmacy, Ootacamund, JSS University, Mysore, India. Email: ksnreddy87@gmail.com

\section{Received date: October 26, 2015}

Accepted date: October 27, 2015

Published date: October 29, 2015

Citation: Karri, V.V.S.R. Importance of selecting route of administration in designing products for Diabetic Foot Ulcers. (2015) J Pharm Pharmaceutics 2(1): 20-21.

\section{Introduction}

Diabetic foot as defined by the World Health Organization (WHO) as, 'The foot of a diabetic patient that has the potential risk of pathologic consequences, including infection, ulceration, and/or destruction of deep tissues associated with neurologic abnormalities, various degrees of peripheral vascular disease, and/or metabolic complications of diabetes in the lower limb ${ }^{[1]}$.

Diabetic foot ulcer (DFU) is a complex pathological phenomenon in which neuropathy and vasculopathy play a vital role and are together termed as neuro-ischemic ulcer. In most of the cases, infection is a consequence of neuro-ischemia and termed as neuro-ischemic ulcers/DFU. Up to $25 \%$ of patients with diabetes develop the corresponding ulcers of the foot. More than half of all foot ulcers become infected, requiring hospitalization, while $20 \%$ of infections result in amputation ${ }^{[2]}$. Diabetes contributes approximately $80 \%$ of all non-traumatic amputations performed every year. After a major amputation, $50 \%$ of people will have the other limb amputated within two years time ${ }^{[3]}$. People with a history of diabetic foot ulcer have a $40 \%$ greater 10 -year death rate than people with diabetes alone. Of the total lower limb amputations, $85 \%$ are due to $\mathrm{DFU}^{[4]}$.

Current DFUs management involves antibiotics, neuropathic drugs, inflammatory modulators, and bioengineered skin substitutes. Many reviews and research studies have discussed the role of a pharmacological agent in treating DFUs but none of studies reports or considered the importance of selecting the route of administration for the application of pharmacological agents. Since DFUs arises of multiple biological pathophysiologies selecting proper route of administration for diverse pharmacological agents is crucial. The significance of route of administration with respect to DFUs treatment is described below.

\section{Topical Therapy}

DFU is associated with vascular diseases; hence, there will be an inadequate supply of blood to the wound site. Hence, oral treatment of DFU may be most likely less effective. Topical therapy has many potential advantages, which includes high local concentration of the drug rather than systemic levels, restriction of total antibiotic usage and respite from the systemic toxicity. Topical treatment for DFU is easier to apply and has fewer side effects compared to other routes of administration. Topical therapies play very important role in the synthesis of therapeutic strategies for DFUs.

\section{Oral Therapy}

Except patient compliance, oral route of administration of drugs will not offer more advantages in treating DFU. Moreover, metabolism of drugs by oral route combined with inadequate blood supply (ischemic ulcers) to wounds leads to supply of dimin- 
ished concentration of drug at the wound site ultimately causing delayed or halted wound healing. Antibiotics and anti-hyperglycemics are the most common drugs administered by this route for the treatment of DFUs

\section{Parenteral Route}

The parenteral route is the most promising route after topical application in treating DFUs. This route offers direct administration of drugs into system circulation and also offers minimal metabolism when compared to the oral route, thereby providing more concentration of drug at the wound site. Antibiotics are the most common drugs used by this route in the treatment of DFUs, since the direct application of antibiotics on wound bed may hurt the wound and may cause lethal effects. Very few drugs are in clinical trials which use the parenteral route.

\section{Intralesional Route}

Intralesional injections have been used to treat a variety of dermatological and non-dermatological diseases. Administration of a drug within or introduced directly into a localized lesion. The purpose of the injection is to attain a high concentration of the drug at the diseased site, with minimal systemic absorption. Compare to all routes, Intralesional route offers potential advantages in delivering drugs or biologiclas to the wound site however, pain involved in this method is the major drawback. Intralesional injection of rhEGF is currently in pipeline for treating DFUs.

\section{Conclusion}

It seems just a matter of time before the research community wakes up to the dangers DFU brings to the table. While designing products for treating DFUs, researchers should not only consider the selection of pharmacological agents but also proper consideration to be given for route of administration also.

\section{References}

1. Bakker, K., Apelqvist, J., Schaper, N.C. International Consensus on the Diabetic Foot and Practical Guidelines on the Management and the Prevention of the Diabetic Foot. (2011) IWGDF.

2. Radhakrishna, K., Karri, V.V.S.N.R., Baskaran, M., Kuppusamy, G. Potential Use of Herbal Medicines in the Treatment of Diabetic Foot Ulcers. (2014) Med Sci 14(56): 34-42.

3. Karri, V.V.S.R., Gowthamarajan, K., Satish Kumar, M.N., Rajkumar, M., Multiple Biological Actions of Curcumin in the Management of Diabetic Foot Ulcer Complications: A Systematic Review. (2015) Trop Med Surg 3:179.

4. Pendsey, S. Reducing Diabetic Foot Problems and Limb Amputation: An Experience from India. (2011) Global Perspective on Diabetic Foot Ulcerations. Croatia 15-24.
Ommega Online Publishers

Journal Title: Journal of Pharmacy \& Pharmaceutics

Journal Short Name: J Pharm Pharmaceutics
Journal ISSN: 2377-1313

E-mail: pharmacoinformatics@ommegaonline.com

Website: www.ommegaonline.org 